\title{
Testicular lymphoma manifesting as neurological symptoms due to secondary neurolymphomatosis: A case report
}

\author{
Nicholas Haslett ${ }^{1}$, Adam Ulano ${ }^{2}$, John DeWitt*3 \\ ${ }^{1}$ University of Vermont Larner College of Medicine, Burlington, VT, USA \\ ${ }^{2}$ Department of Radiology, The University of Vermont Medical Center, Burlington, VT, USA \\ ${ }^{3}$ Department of Pathology and Laboratory Medicine, The University of Vermont Medical Center, Burlington, VT, USA
}

Received: August 21, 2020

Accepted: December 15, 2020

Online Published: January 27, 2021

DOI: $10.5430 /$ crcp.v8n1p1

URL: https://doi.org/10.5430/crcp.v8n1p1

\begin{abstract}
Neurolymphomatosis is a rare manifestation of lymphoma presenting as diffuse invasion and involvement of peripheral and spinal nerves. Due to the common presenting symptomatology of neurologic complaints localizing to the affected peripheral nerve, lymphoma as the underlying etiology can be difficult to diagnose. Here we present the case of a gentleman presenting with right extremity neuropathic symptoms, subsequently discovered to have diffuse large B-cell lymphoma of testiticular orgin after nerve biopsy revealed neurolymphomatous involvement of a spinal nerve. This case highlights the importance of the consideration of neurolymphomatosis in the work up of neuropathic symptoms, as well as the full assessment for the site of primary involvement.
\end{abstract}

Key Words: Neurolymphomatosis, Lymphoma, DLBCL, Neuro-oncology

\section{INTRODUCTION}

Neurolymphomatosis (NL) is a rarely diagnosed disease process marked by lymphomatous invasion of the cranial or peripheral nerves in the setting, most commonly, of diffuse large B-cell lymphoma and more rarely in leukemia, ${ }^{[1,2]}$ or other lymphomas, ${ }^{[3]}$ with an estimated prevalence of $0.2 \%$ in non-Hodgkin lymphoma. ${ }^{[4]}$ The presentation of NL varies, and can involve weakness, sensory changes, areflexia, or sudden pain, ${ }^{[5]}$ with a wide differential ranging from peripheral nerve sheath tumor to demyelinating disease depending on presentation, and therefore requires a high degree of suspicion to make the diagnosis.

\section{Case presentation}

Here we present the case of a 76-year-old man with a past medical history of hyperlipidemia and degenerative disc disease who presented with a two-year history of right foot pain with numbness and a ball-like feeling on the bottom of his foot. He also described a one-year history of dysesthetic pain in the left anterior thigh that improved on its own over the course of three months. He reported that, two months before presenting to neurology, he experienced severe pain in the right lower back with radiation to the left buttock, followed several weeks later by severe pain down the right leg in the L4 distribution. His right leg began giving out so he could not walk any distance and he was using a wheelchair when he presented. His physical exam was significant for some

\footnotetext{
* Correspondence: John DeWitt; Email: John.dewitt@uvmhealth.org; Address: Department of Pathology and Laboratory Medicine, MP1-104, The University of Vermont Medical Center, 111 Colchester Ave, Burlington, VT, 05401, USA.
} 
weakness of anterior tibialis and extensor hallux bilaterally, the left side weaker than the right. There was also decreased sensation to light touch throughout the right lower leg and dorsum of the right foot. The patient has no known risk factors or family history of lymphoma.

MRI revealed enlargement of right L4 nerve root (see Fig- ures $1 \mathrm{~A}-1 \mathrm{C})$. The patient had a lumbar MRI 6 months earlier, which had shown comparatively normal-sized L4 nerve (see Figure 1D). In light of a normal-appearing nerve six months previously, multiple nerve involvement, and diffuse abnormal enhancement, the differential in this case prior to biopsy included infectious, inflammatory, and neoplastic conditions with lymphoma the leading consideration.
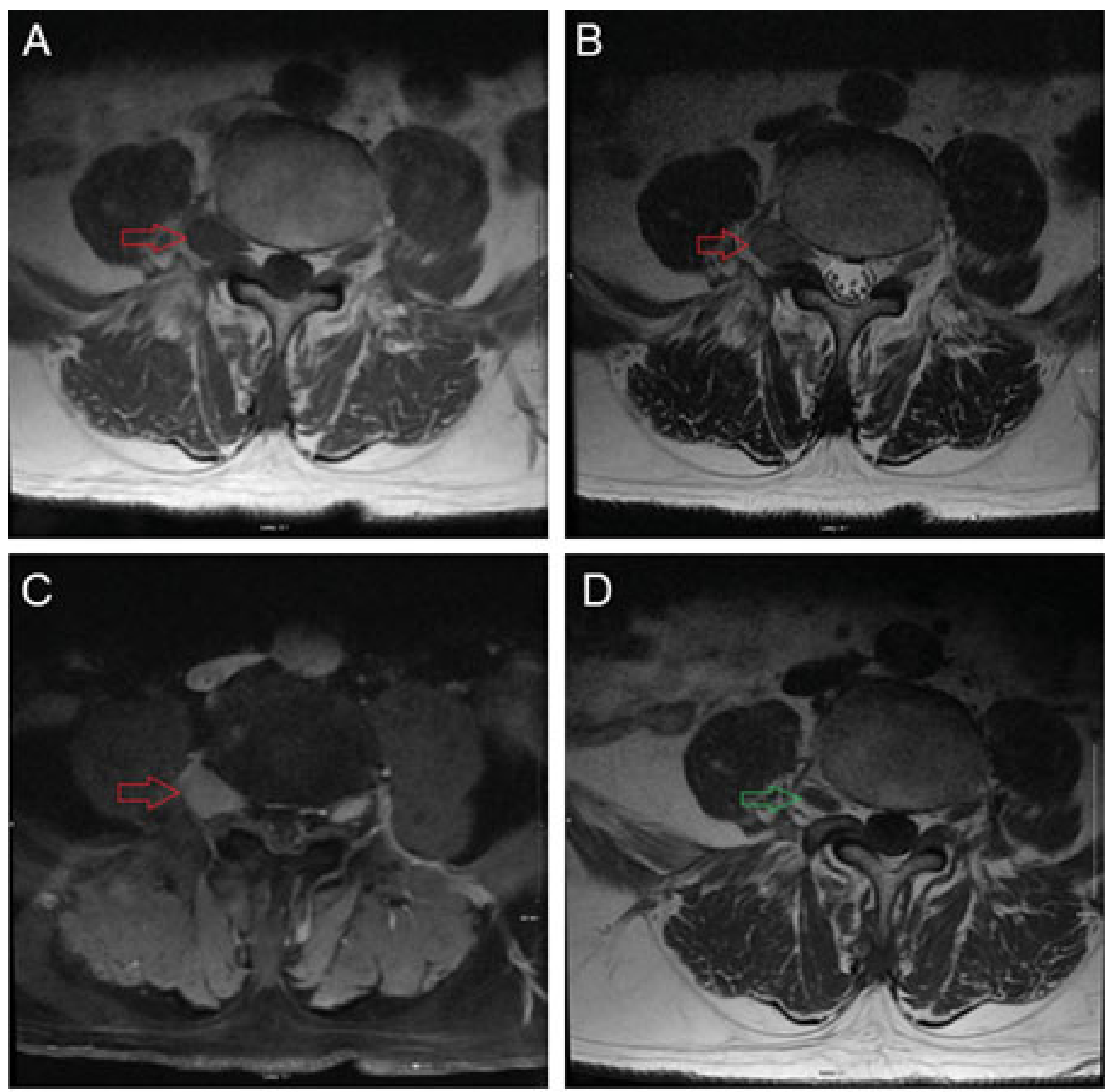

Figure 1. Radiologic findings. Axial, T1-weighted MR image of the lower lumbar spine at the level of the L4 inferior endplate (A) demonstrates abnormal fusiform enlargement of the right L4 nerve in the foraminal and extra-foraminal zones (red arrow). Axial, T2-weighted MR image at the same level (B) demonstrates the enlarged nerve (red arrow) which was clearly separate from the adjacent intervertebral disc (not shown). Axial, T1-weighted, contrast-enhanced, fat-suppressed MR image at the same level (C) shows corresponding abnormal, homogeneous enhancement of the enlarged nerve (red arrow). Axial, T1-weighted MR image of the lower lumbar spine at the same level (D) obtained six months previously demonstrates a normal-sized right L4 nerve (green arrow). 

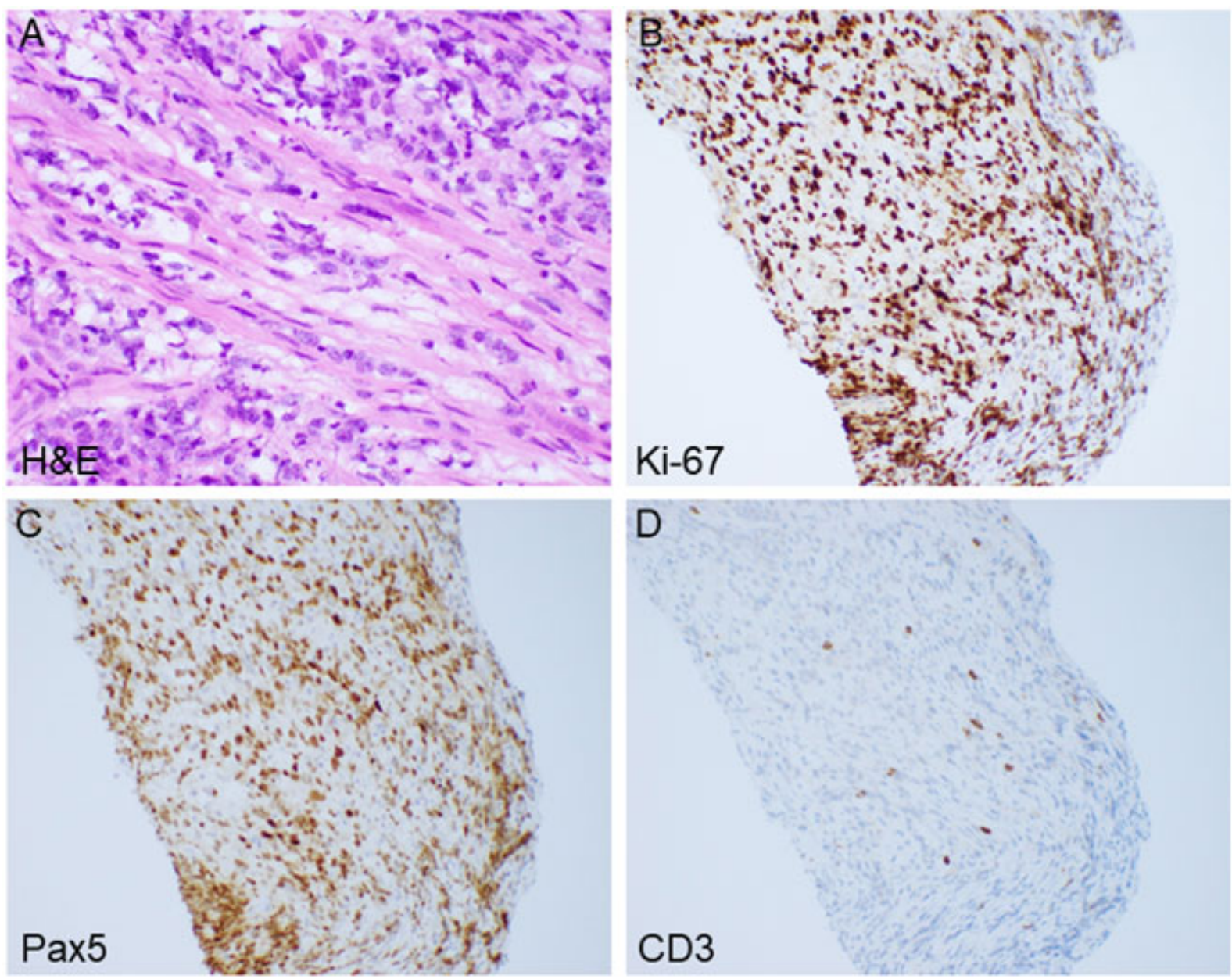

Figure 2. H\&E stained section (60x) shows large atypical lymphoid cells involving peripheral nerve (A). Ki-67 immunohistochemistry reveals a highly elevated proliferation index (B). Pax5 shows the atypical infiltrate consists mainly of B-cells (C), and scattered CD3-positive T-cells (D).

The nerve was biopsied, and frozen section showed diffuse infiltrate of large atypical lymphoid cells with irregular to lobated nuclei, and scant to moderate quantity of pale cytoplasm infiltrating among nerve axons. Immunohistochemistry showed the infiltrate consisted mostly of B-cells $(\mathrm{CD} 20+/ \mathrm{Pax} 5+)$ and scattered T-cells (CD3+)(see Figures 2A-2D).

Additional immunohistochemistry demonstrated that the large atypical cells co-expressed Bc16, Bcl2, and Mum1, and were largely negative for CD10 and cyclinD1. C-myc was positive in $20 \%$ of tumor cells. In situ hybridization showed neoplastic cells were EBER negative. Ki-67 showed a proliferation index of $60 \%-70 \%$.

The histologic and immunophenotypic features together supported a diagnosis of diffuse large B-cell lymphoma most consistent with non-germinal center, activated B-cell origin with diffuse infiltration of spinal nerve roots consistent with neurolymphomatosis. Intrathecal administration of high- dose methotrexate with concomitant cerebral spinal fluid (CSF) analysis was initiated. After the first administration of methotrexate, the patient experienced acute kidney injury (AKI), and so administration was stopped. CSF at no point yielded malignant cells, but did show nucleated cells $(1,407$ cells/cmm), elevated protein $(363 \mathrm{mg} / \mathrm{dL})$, normal glucose, and elevated neutrophils. Further work-up involved whole body PET CT, which revealed diffuse radiotracer uptake in the right testicle and focal, significant radiotracer uptake in the left testicle. An ultrasound confirmed the presence of bilateral testicular masses, and subsequent bilateral orchiectomy confirmed testicular involvement by diffuse large B-cell lymphoma. After orchiectomy, treatment with MR-CHOP (methotrexate, rituximab, cyclophosphamide, doxorubicin, vincristine, and prednisone) was planned, but this regimen was never begun owing to the AKI after initial administration of intrathecal methotrexate. An Ommaya reservoir was placed, but post-op imaging revealed the device to be malpositioned. The patient subsequently developed significant 
right frontal hemorrhage after the procedure, with deteriorating neurological exam involving hemiparesis and flat affect. Head venogram at that time showed severed vessels, and the patient was transferred to SICU for cerebral edema with high-dose steroids and hypertonic saline, at which time the hemorrhage appeared stable on repeat imaging. The Ommaya was removed, and the patient experienced improved neurological status. A second PET scan demonstrated progression of disease to right lower cervical, mediastinal, and right hilar lymph nodes as well as multiple lumbar and sacral nerve roots. The patient received palliative radiation, was discharged to a palliative care facility, and passed away several days later.

Multiple entities can cause abnormal nerve enhancement on MRI, as seen in this case. The differential for enhancement is somewhat dependent upon whether the enhancement involves a single nerve or multiple. Generally speaking, enhancement of a single nerve or a few nerves may be encountered in the setting of reactive neuritis or in the postoperative setting due to intraoperative stretching of the nerve. Certain infectious (e.g. HIV radiculopathy, Lyme disease), inflammatory (e.g. sarcoidosis), demyelinating (e.g. GBS, CIDP), and neoplastic (e.g. metastatic disease, lymphoma) conditions may cause enhancement of a solitary nerve but diffuse involvement is more common. Diffuse enlargement of the nerves without abnormal enhancement may be encountered in the setting of CIDP or hereditary motor-sensory

\section{REFERENCES}

[1] Baehring JM, Damek D, Martin EC, et al. Neurolymphomatosis. Neuro Oncol. 2003; 5: 104-115. PMid:12672282. https://doi . org/10.1215/15228517-5-2-104

[2] Grisariu S, Avni B, Batchelor TT, et al. Neurolymphomatosis: An International Primary CNS Lymphoma Collaborative Group report. Blood. 2010; 115: 5005-11. PMid:20368468. https://doi.org/ 10.1182/blood-2009-12-258210

[3] Roberts M, Chan A. A Rare Case of Primary Neurolymphomatosis in Natural Killer T-Cell Lymphoma. Neurology. Apr 2019; 92 (15 Supplement): P2.6-005.

[4] Avila JD, Vivar C. Neurolymphomatosis: A review of 82 cases. Neurology. Apr 2017; 88 (16 Supplement): P5.141. https://doi.or g/10.1016/S1474-4422(16) 30350-7

[5] Shree R, Goyal MK, Modi M, et al. The diagnostic dilemma of neurolymphomatosis. J Clin Neurol. 2016; 12(3): 274-281. neuropathies (e.g. Charcot-Marie-Tooth). Plexiform neurofibromas in the setting of Neurofibromatosis typically demonstrate the classic appearance of nerve enlargement by tumor without significant enhancement. Occasionally, extruded disc material may extend along a nerve in the extra-foraminal zone but extruded disc material does not typically enhance.

\section{DiscuSSION}

Solid organ primary diffuse large B-cell lymphoma rarely presents with signs and symptoms of neurolymphomatosis, including, to our knowledge, only two previous cases in the literature in which a testicular lymphoma was discovered after a patient presented with a set of neurological symptoms resulting from a secondary neurolymphomatosis. ${ }^{[6,7]}$ In a previous review of the literature highlighting 23 cases of NL involving the lumbar / sacral nerves, nerve involvement was the primary finding, without evidence of solid organ involvement by lymphoma. ${ }^{[8]}$ Additionally, in another larger series, only 11 of 72 patients diagnosed with NL were found to have concurrent systemic involvement by lymphoma. ${ }^{[1]}$ Cases such as the one presented here highlight the importance of tissue diagnosis when a rare entity such as neurolymphomatosis is entertained, as well as a full systemic work up in suspected cases, as the neurologic manifestations may represent the cardinal sign of an undeclared occult lymphoma.

\section{CONFLicts OF InTEREST Disclosure}

There is no conflict of interest.
PMid:27449910. https://doi.org/10.3988/jcn.2016.12.3. 274

[6] Sunami Y, Gotoh A, Hamano Y, et al. Various neurological symptoms by neurolymphomatosis as the initial presentation of primary testicular lymphoma. Case Rep Oncol. 2015; 8(1): 200-204. PMid:26034480. https://doi .org/10.1159/000381874

[7] Facchinelli D, Ciliberti E, Stussi G, et al. Sciatic pain by neurolymphomatosis as initial presentation of disseminated diffuse large B cell lymphoma involving the testis and the CNS. Hematological Oncology. 2020; 38(2): 197-200. PMid:32026488. https: //doi.org/10.1002/hon.2698

[8] Bourque PR, Sampaio ML, Warman-Chardon J, et al. Neurolymphomatosis of the lumbosacral plexus and its branches: case series and literature review. BMC Cancer. 2019; 19(1): 1149. Published 2019 Nov 27. PMid:31775683. https://doi.org/10.1186/s1 2885-019-6365-y 